\title{
Evaluation of Inverse Problem with Slow-Conducting Channel in Scar Area in a Post-Infarction Model
}

\author{
Zexi Chen ${ }^{1}$, Miguel Rodrigo ${ }^{2}$, Alejandro Liberos ${ }^{3}$, Ismael Hernandez-Romero ${ }^{3}$, \\ Jesus Requena ${ }^{1}$, Andreu M Climent ${ }^{2,3}$, Maria S Guillem ${ }^{2}$ \\ ${ }^{1}$ Queen Mary University of London, London, UK \\ ${ }^{2}$ Instituto ITACA, Universitat Politècnica de València, Valencia, Spain \\ ${ }^{3}$ CIBERCV, Hospital General Universitario Gregorio Marañón, Instituto \\ de investigación sanitaria Gregorio Marañón, Madrid, Spain
}

\begin{abstract}
Background: Slow-conducting channels in postinfarction scar areas constitute a life-threatening substrate, since they may lead to reentries and further fibrillation. Inverse problem methods may help to noninvasively locate these channels.

Methods: Cardiac activity during chronic infarction was simulated based on Bueno-Orovio's cardiac cell model on a mesh representing a realistic ventricular anatomy. Ventricular electrograms (EGMs) were computed and used to obtain the torso surface electrical activity. The inverse problem resolution with zero-order Tikhonov regularization was used to calculate the inverse EGMs on the ventricular epicardial surface. Activationrecovery intervals (ARIs), defined as the interval between times of minimum derivative of $Q R S$ and maximum derivative of $T$ wave, were measured from the departing and inverse-computed EGMs and subsequently compared.

Results: ARIs were shorter in the channel than in the border zone and healthy area, both in the simulated EGMs and in their inverse-computed counterparts.

Conclusion: The results in this paper show that the channel inside a post infarction scar zone can be noninvasively identified by using inverse problem approaches.
\end{abstract}

\section{Introduction}

The rates of ischemic heart disease (IHD) mortality appear to be accelerated in this decade. Among these IHDs, slow-conducting channels in post-infarction scar areas constitute one of the most dangerous situations, since they may lead to re-entries and further fibrillation [1]. A specific challenge is to identify the presence of these channels and determine whether to give intervention to the patient to close the channel.

The inverse problem allows the reconstruction of the cardiac activity non-invasively, by which the cardiac potentials are mathematically related with the electric potentials on the torso. In this context, a post-infarction human ventricular model is a suitable platform to integrate the slow-conducting channels inside scar area conditions and to simulate the body surface potential mapping and recover the heart electrical activity by means of the inverse problem resolution. The aim of this work is to evaluate the inverse problem as a non-invasive diagnosis tool of slow-conducting channels in infarcted hearts.

\section{Methods}

\subsection{Anatomical Heart-Torso model}

A torso geometrical model established by MacLeod et al. [2], which consisted of 771 nodes and 1538 tetrahedrons, was used to present the electrical activity on the torso. The ventricular model used was composed by 155,936 nodes and 899,243 tetrahedrons, and the cellular mathematical model was simulated for each of node. A human ventricular mesh, located inside the torso volume according to anatomy, was $1 \mathrm{~mm}$ away from the heart torso and generated from the computer software.

\subsection{Electrophysiology and tissue model}

We performed a computational simulation for human ventricular electrical propagation with: (i) a myocardium model based on the ventricular action potential model of Bueno-Orovio et al. [3]; (ii) electrical remodelling in the border zone area by reducing the conduction velocity in $30 \%$, uplifting the resting potential in $10 \%$ and extending the Action Potential Duration (APD) in $30 \%$. 
Specifically, the $\mathrm{APD}_{90}$ was mainly used for the measurement of the APDs, which was measured as the time interval between the $90 \%$ of the voltage at the upstroke and at the down stroke of the APs. The tissue activation was mainly in accordance with the human ventricular activation sequence of Cardone-Noott et al. [4], which the left ventricle (LV) endocardial surface was activated within $30 \mathrm{~ms}$ and the transmural propagation accomplished in $35 \mathrm{~ms}$. As illustrated in the Tables 1-3, the model parameter values were modified to accommodate post-infarction condition for epicardial, endocardial and midmyocardial myocytes.

Table 1. Model parameter modification of epicardial myocytes for APDs extension. [5]

\begin{tabular}{llll}
\hline & Healthy & Border & Scar \\
\hline$k_{w}^{-}$ & 65 & 65.0356 & 65.0356 \\
$\tau_{o 1}$ & 400 & 6.3196 & 5.3307 \\
$\tau_{s o 1}$ & 30.0181 & 56.6591 & 56.1802 \\
$\tau_{s o 2}$ & 0.9957 & 1.0809 & 1.5291 \\
$k_{s o}$ & 2.0458 & 2.2695 & 2.4552 \\
$\tau_{s 2}$ & 16 & 7.3099 & 7.6184 \\
$k_{s}$ & 2.0994 & 5.6456 & 5.7472 \\
\hline
\end{tabular}

Table 2. Model parameter modification of endocardial myocytes for APDs extension.

\begin{tabular}{clll}
\hline & Healthy & Border & Scar \\
\hline$k_{w}^{-}$ & 200 & 201 & 201 \\
$\tau_{o 1}$ & 470 & 7.4256 & 6.2636 \\
$\tau_{s o 1}$ & 40 & 6 & 56.1802 \\
$\tau_{s o 2}$ & 1.2 & 1.3027 & 1.8597 \\
$k_{s o}$ & 2 & 2.2187 & 2.4002 \\
$\tau_{s 2}$ & 2 & 0.9137 & 0.9523 \\
$k_{s}$ & 2.0994 & 2.0994 & 5.7472 \\
\hline
\end{tabular}

Table 3. Model parameter modification of midmyocardial myocytes for APDs extension.

\begin{tabular}{llll}
\hline & Healthy & Border & Scar \\
\hline$k_{w}^{-}$ & 200 & 201 & 201 \\
$\tau_{o 1}$ & 410 & 6.4776 & 5.4640 \\
$\tau_{s o 1}$ & 91 & 400 & 400 \\
$\tau_{s o 2}$ & 0.8 & 0.8685 & 1.2398 \\
$k_{s o}$ & 2.1 & 2.3296 & 2.5202 \\
$\tau_{s 2}$ & 4 & 1.8275 & 1.9047 \\
$k_{s}$ & 2.0994 & 2.0994 & 5.7472 \\
\hline
\end{tabular}

Ventricular EGMs were calculated from the electrical potential on the ventricular surface and then used to obtain the electrocardiograph (ECG) on the torso with a forward problem propagation. A corresponding inverse problem method, implemented by using BEM method and zero-order Tikhonov regularization, with election of the regularization parameter by using the L-curve method, to calculate the inverse EGMs on the ventricular mesh. [6] The activation-recovery intervals (ARIs), previously defined by Haws et al. [7] as the time interval between the minimum time derivative of QRS wave and maximum derivative of $\mathrm{T}$ wave, were measured from the EGMs and the inverse-computed EGMs.

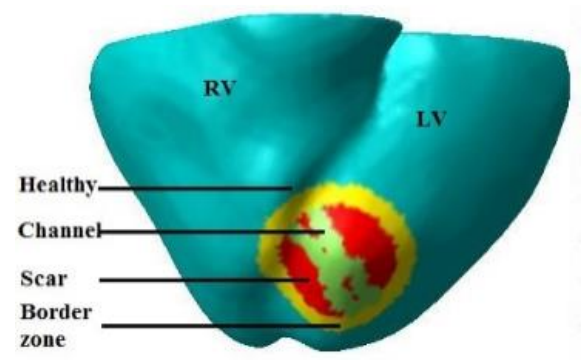

Figure 1 Definition of post-infarction human ventricle.

\subsection{Post-Infarction Pattern Design}

We designed a slow-conducting channel inside scar area pattern based on experimental appearance of acute myocardial infarction in pigs. [8] A nummular area including the scar, channel and border zone area was located in the anterior wall of the left ventricle (LV). The scar and border zone area penetrated the front left ventricle, while the channel only exists on the surface of the front left ventricle. As clarified in the Figure 1, a scar area (Red) of $7.2 \mathrm{~cm}^{2}$ was surrounded with a border zone (Yellow) of $5.4 \mathrm{~cm}^{2}$ and with a slow-conducting channel (Green) of $3.2 \mathrm{~cm}^{2}$ and $1.2 \mathrm{~cm}$ width. The myocytes inside the scar area cannot be activated during the electrical propagation, while the myocytes in the border zone and channel area had slow conduction velocity.

\section{Results}

\subsection{Model Construction and Validation}

Slow-conducting channel inside the scar area in a ventricular post-infarction condition exhibited a few distinct characteristics: (i) longer APDs in border zone area, (ii) slower conduction velocity inside the channel, (iii) inactivation of dead scar area, which were included during the model construction.

Consecutive transmembrane potential maps in Figure 2 indicate the electric propagation through the ventricle (except the scar area) from several earliest activation sites, the inactivation of the myocytes in scar area, and the conduction of the channel inside the scar area. 
Measured activation times for post-infarction condition are presented in Figure 3. The dark red corresponded to the non-activated scar area, meanwhile, the sudden colour change from dark blue to light blue validating the slower conduction velocity in the channel area.
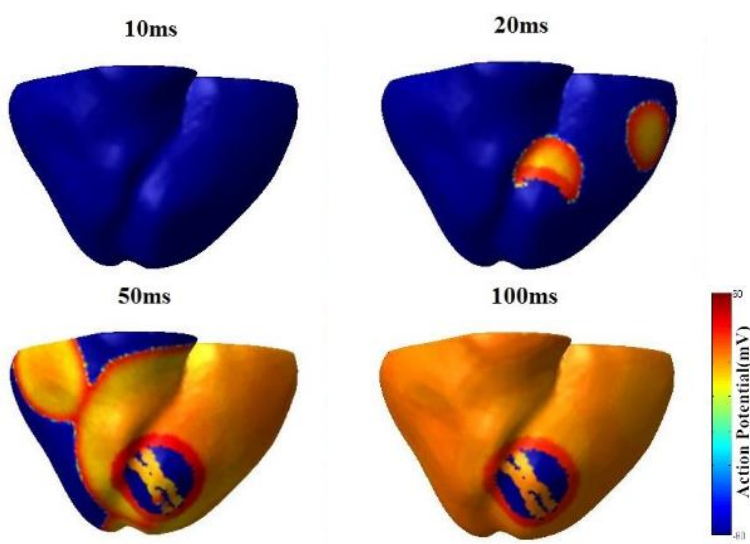

Figure 2 Isopotentials at $10 \mathrm{~ms}, 20 \mathrm{~ms}, 50 \mathrm{~ms}$ and $100 \mathrm{~ms}$ respectively.

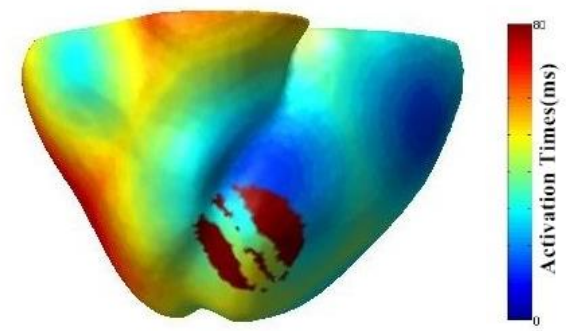

Figure 3 Anterior views of epicardial activation times.

APDs for ventricular post-infarction model are shown in Figure 4 for the confirmation of the extension of APDs in the border zone area. The simulated action potential duration was $315 \mathrm{~ms}, 385 \mathrm{~ms}$, and $200 \mathrm{~ms}$ for the healthy, border zone and channel areas respectively, and the conduction velocity $(\mathrm{CV})$ was $364 \mathrm{~cm} / \mathrm{s}, 168 \mathrm{~cm} / \mathrm{s}$ and $163 \mathrm{~cm} / \mathrm{s}$, respectively.

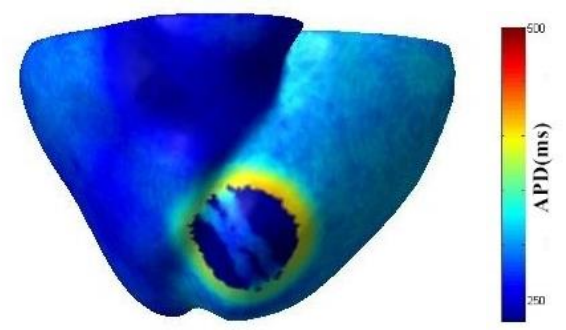

Figure 4 Anterior views of epicardial APDs.

Samples of the EGMs and inverse EGMs from each area are provided in Figure 5 to show the disparity after the inverse problem method.

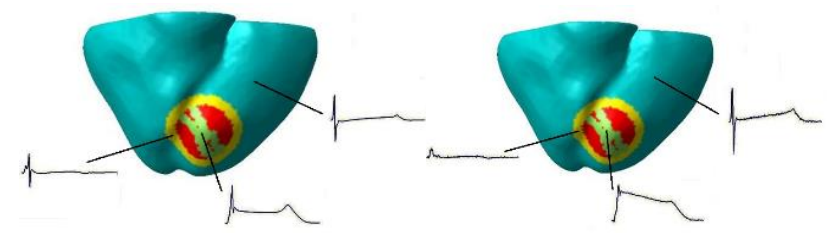

Figure 5 Samples of EGM and inverse EGM from border zone, channel and healthy area respectively.

\subsection{Correlation between ARIs of EGMs and APD 90}

As mentioned earlier, the correlation between the ARIs and APDs allowing us to measure ARIs from the departing EGMs and inverse EGMs to equally represent APDs. The linear regression plot of relation between correlations between ARIs of EGMs and $\mathrm{APD}_{90}$ (Figure 6) during post-infarction period assured the equivalence between ARIs of EGMs and $\mathrm{APD}_{90}$ in this study $(\mathrm{r}=$ 0.99).

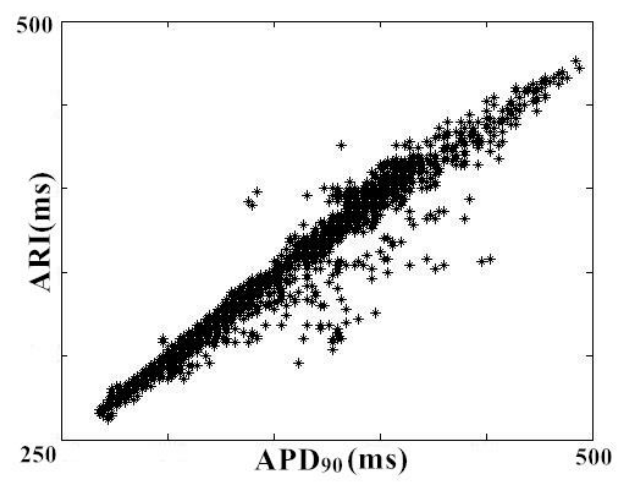

Figure 6 Correlations between activation times of EGMs and action potentials, and correlations between ARIs of EGMs and $\mathrm{APD}_{90}$.

\subsection{Comparison of ARIs between EGMs and inverse EGMs}

Schematic views of the ventricular ARIs were provided in Figure 7 for ease of comparison of ARIs between EGMs and inverse EGMs. The ARIs of departing EGMs were shorter in the channel (332 \pm 17 $\mathrm{ms})$ than in the border zone $(425 \pm 34 \mathrm{~ms})$ and healthy area $(361 \pm 39 \mathrm{~ms})$. Inverse-computed EGMs showed the same trend $(320 \pm 37 \mathrm{~ms}$ for channel, $391 \pm 49 \mathrm{~s}$ for border zone and $358 \pm 51 \mathrm{~ms}$ for healthy tissue). Due to the correlation between the ARIs and APDs, when the APDs were extended in the mathematical model, the ARIs would be also extended in the border zone area in the EGMs and inverse EGMs respectively. This feature of 
channel areas allowed identifying the slow-conduction channel with width of $1.57 \mathrm{~cm}$ from departing EGMs and with a width of $1.78 \mathrm{~cm}$ from inverse-computed EGMs at its correct position.
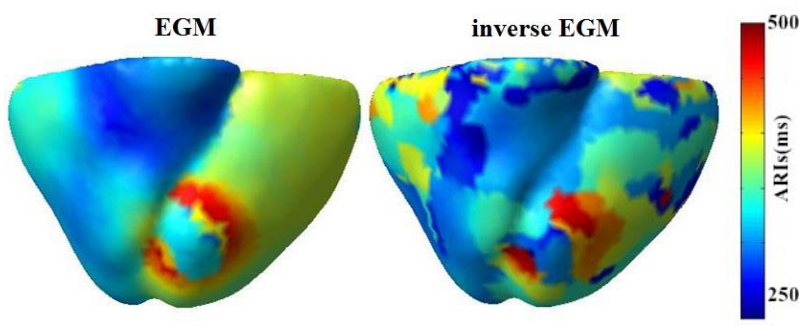

Figure 7 Comparison of ARIs between EGMs and inverse EGMs.

Table 4 Measured APDs, CV and ARIs.

\begin{tabular}{llll}
\hline & Healthy & Border & Channel \\
\hline APDs $(\mathrm{ms})$ & 315 & 385 & 200 \\
$\mathrm{CV}(\mathrm{cm} / \mathrm{s})$ & 364 & 168 & 163 \\
ARIs of EGMs(ms) & $361 \pm 39$ & $425 \pm 34$ & $332 \pm$ \\
& & & 17 \\
ARIs of Inverse EGMs & $358 \pm 51$ & $391 \pm 49$ & $320 \pm 37$ \\
\hline
\end{tabular}

\section{Discussion}

This mathematical study presents a simulation using a simple model to justify the use of inverse problem approach as method for electroanatomical reconstruction. By building up the specific mathematical model, we include the chronic post-infarction condition in a realistic ventricular anatomy to simulate the cardiac activity. With the EGMs and inverse EGMs, the ARIs, on behalf of the APDs, are measured and compared to locate the slowconduction channel and evaluate the performance of the inverse problem approach. Since the ARIs successfully help to differentiate the scar and channel area, we conclude that the slow-conduction channel inside a post infarction scar zone can be non-invasively identified by using inverse problem approaches.

The inverse problem method presented here have been shown to be an effective approach to recover the main characteristics of the human ventricular chronic infarction. However, the underlying simplifications on mathematical model may limit the accuracy of subordinate characteristics. Though the APDs were carefully measured, the CV was not measured for each cellular node. In addition, due to the lack of APDs data in human midmyocardial and endocardial myocytes, the modifications on these two kinds comply with the same trend considering the epicardial myocytes.

\section{References}

[1] Vergara, P., Roque, C., Oloriz, T., Mazzone, P., Bella, P.D. Substrate mapping strategies for successful ablation of ventricular tachycardia: A review. Arch Cardiol Mex 2013;83(2):104-111

[2] MacLeod, R.S., Gardner M., Miller, R.M., Horácek B.M. Application of an electrocardiographic inverse solution to localize ischemia during coronary angioplasty. J Cardiovasc Electrophysiol 1995; 6: 2-18

[3] Bueno-Orovio, A., Cherry, E.M., Fenton, F.H. Minimal model of human ventricular action potentials in tissue. Journal of Theoretical Biology 2008; 253:544-560

[4] Cardone-Noott, L., Bueno-Orovio, A., Minchole, A., Zemzemi, N., Rodriguez, B. Human ventricular activation sequence and the simulation of the electrocardiographic QRS complex and its variability in healthy and intraventricular block conditions. Europace. 2016; 18: 4-15

[5] Lassila, T., Lange, M., Porras Perez, R.A., Lekadir, K., Alba, X., Piella, G., Frangi, F.A. Electrophysiology model for a human heart with ischemic scar and realistic purkinje network. In: Oscar, C. et al., editor. Statistical Atlases and Computational Models of the Heart: Imaging and Modelling Challenges. 2016:90-97

[6] Rodrigo M., Climent, A.M., Liberos, A., Fernández-Aviles, F., Berenfeld, O., Atienza, F., Guillem, M.S. Highest dominant frequency and rotor positions are robust markers of driver location during non-invasive mapping of atrial fibrillation: A computational study. Heart Rhythm. 2017; 14:1224-1233.

[7] Haws, W.C., Lux, L.R. Correlation between in vivo transmembrane action potential durations and activationrecovery intervals from electrograms. Circulation 1990; $81: 282-288$

[8] Arenal, A. Esther Pérez-David, MD, Pablo Ávila, MD, Javier Fernández-Portales, MD, Verónica Crisóstomo, DVM, PhD, Claudia Báez, DVM, Javier Jiménez-Candil, $\mathrm{MD}, \mathrm{PhD}$, José L. Rubio-Guivernau, PhD, María J. Ledesma-Carbayo, PhD, Gerard Loughlin, MD, Javier Bermejo, MD, PhD, Francisco M. Sánchez-Margallo, $\mathrm{DVM}, \mathrm{PhD}$, Francisco Fernández-Avilés, $\mathrm{MD}, \mathrm{PhD}$. Noninvasive identification of epicardial ventricular tachycardia substrate by magnetic resonance-based signal intensity mapping. Heart Rhythm. 2014;11:1456-64

Address for correspondence.

Zexi Chen

University of California, San Diego, La Jolla, CA 92093.

zec058@eng.ucsd.edu 